\title{
SM-SIP: Seamless Mobility Management for Heterogeneous Wireless Networks
}

\author{
Khaled Zeraoulia \\ University of Sciences and Technology Houari Boumediene (USTHB) El Alia BP 32, Bab Ezzouar 16111 \\ Tel/ Fax: 213021247187 Algiers, Algeria \\ khaled.zeraoulia@gmail.com \\ Nadjib Badache \\ Research Center on Scientific and Technical Information CERIST Algiers, Algeria \\ nbadache@Cerist.dz
}

\begin{abstract}
Next-generation wireless networks (NGN) and internet of Thing (IoT) become two leaders of designing mobile multimedia services. The deployment of these services using heterogeneous wireless networks will be the important factor that leads to profound change in the way that these services are delivered. Also, how these services will be managed is a real challenge. Consequently, Seamless mobility management should be provided to support various services in heterogeneous networks. A SIP-based network appears as a very attractive alternative to mobile multimedia applications. In this paper, we introduce a novel mobility management strategy for mobile SIP networks, in which we develop a seamless handover used mobile SIP scheme called SMSIP (Seamless Mobility Management for Heterogeneous Wireless Networks for Mobile SIP environment). SM-SIP can significantly reduce the system signaling cost and handover delay, by proactively processing the address allocation and session updates using link layer information of wireless networks. Also, our performance study shows that SM-SIP reduces efficiently packets loss using an anticipated buffering scheme.
\end{abstract}

Index Terms - Seamless handoff, Fast handoff, Multimedia applications, QoS, Hierarchical SIP, Mobility management, Wireless communication).

\section{INTRODUCTION}

In the last few years the number of mobile devices as well as access technologies has increased and the number devices are expected to increase with growing speed [1]. In addition, the rise of these devices in a communicating network creates the Internet of Things (IoT) which is the next revolutionary technology in transforming the internet into a fully integrated future internet [2].

This new concept will provide new services and ubiquitous communications including large numbers of nodes (PDAs, laptops, sensors, etc.) with varying functionalities and carried by heterogeneous wireless networks (HWNs) [3][4][26]. So, designing these new services is a real challenge to overcome. In this work, we focus on the mobility management of these services in heterogeneous wireless networks (Fig.1).

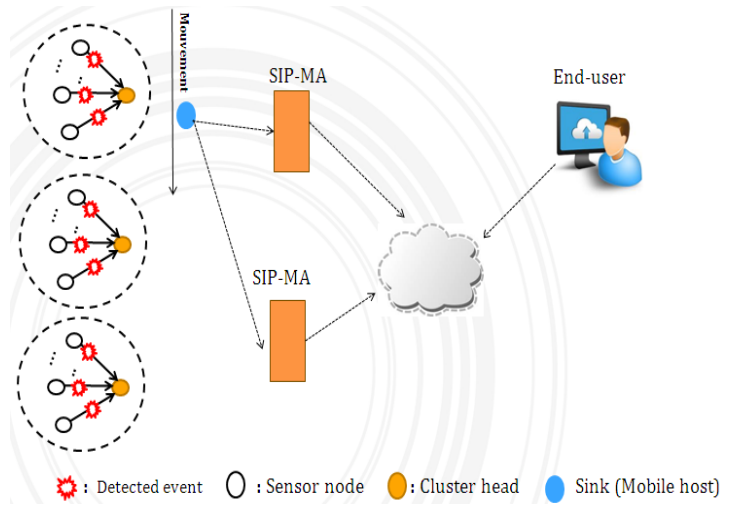

Fig.1. SM-SIP application

In our mobile multimedia service design, we assume that we have three important components: the first is the wireless sensing networks formed by the wireless sensor nodes. The second is cluster head in which a node serves as cluster head $(\mathrm{CH})$ that buffer data originated from sources and transfer to sink nodes when they arrive. $\mathrm{CH}$ enables sink node to collect a large volume of data at a time without traveling long distances, Sink node moves along sensing areas and transmits sensing information to the third component which is the end- user which in turn may send information to the sink node. In this context, the designer of these services must investigate the mobility of sink node to meet QoS requirements. In mobile networks, mobile multimedia applications are delay-sensitive, loss-sensitive and causing overhead. There are mainly four ways of providing mobility nowadays. The first approach concerns link-layer mobility that is specific for each radio access network. In cellular networks, the handover from one node to another one is performed with the serving radio network subsystem relocation procedure [5][29]. The second approach handles mobility at the network layer by using Mobile IP [6]. The third approach is to consider mobility at the transport layer by using mobile SCTP (Stream Control Transmission Protocol) [7]. The last approach is 
to use application-layer mobility with Session Initiation Protocol (SIP) [8].

Although the mobile SIP and mobile IPv6 [9][10] offer mobility management capability, mobile IPv6 is not suitable for supporting streaming media with stringent delay and eliminates packet losses requirements. Enabling mobility in IP networks is an important issue for making use of the many light-weight devices appearing at the market. The IP mobility support being standardized in the IETF uses tunnelling of IP packets from a Home Agent to a Foreign Agent to make the mobility transparent to the higher layer. There are a number of problems associated with Mobile IP, such as routing, each host needing a home IP address, tunnelling management, etc. furthermore, Future mobile systems, user demand for continuous connectivity is increasing irrespective of which type of interface or network they are using. With the growth of the wireless internet and the mobile computing marketplace we expect to see mobility without any geographical or network boundaries.

In addition, several works in littirature investigated allIP communication between WSN and IPv6 networks [11][12], such as IPv6 over Low power Wireless Personal Area Networks (6Lo-WPAN)[13] which is a protocol architecture that has recently been ratified to enable the seamless interconnectionof wireless sensor networks (WSN) to the Internet. Although, these works bring significant impact especially for interconnection way and data transmission, these improvements remain insufficient against flexibility, address configuration, Mobility, routing mechanisme and congestion problem.

In this situation, the mobility management needs careful investigation to offer reasonable delays and packet loss for mobile multimedia and real time applications in different type of network. Mobile-SIP is evolving as the dominant protocol for multimedia call control in wireless and mobile networks. Providing seamless mobility support is one of the most challenging problems towards the system integration of fourth generation (4G) wireless networks [14]. Most of the related literature focuses on seamless mobility aiming to reduce the packet loss rate and handover delay which would eventually lead to efficiency mobile multimedia communication. Also, application-layer mobility management protocol like the Session Initiation Protocol (SIP) has been considered as the right candidate for handling mobility in the heterogeneous $4 \mathrm{G}$ wireless networks.

Several contributions have been proposed to improve performance during handover [15] [16] [17]. Handover latency is the primary cause of packet loss resulting in performance degradation of Mobile IPv6 or mobile SIP. Mobile IPv6 with fast Handover enables a Mobile Node $(\mathrm{MN})$ to quickly detect at the IP layer that it has moved to a new subnet by receiving link-related information from the link-layer [18]. Several research have been conducted in order to improve Link layer handoff performance in terms of handoff delays and packet loss rates for fast handover transmission. Since probe delays consist of the main contributor to the overall L2 handoff latency. [19] proposed a new intelligent fast handover scheme for mobile IPv6-based WLANs. The new approach consists of equipping mobile nodes with pre-configured mobility pattern to select new access point prior to attachment. [20] conducted a simulative evaluation of the overall performance of Fast Handovers for Mobile IPv6 in comparison. [21] enhanced buffer management scheme for Fast handover protocol. [22] described a fast handover scheme based mobile IP ( FMIPv6) combining two operation modes is analyzed considering the PPMF that is affected by the radius of a cell, velocity of mobile nodes, and the layer 2 triggering time. The effect of system parameters, such as the PPMF, the time required to process additional layer 3 signaling, and the layer 2 trigger time, is analytically investigated with respect to the signaling cost and the packet delivery cost. [18] proposed an enhancement to Fast Mobile IPv6 handover (FMIPv6), based on link layer information. Also, we proposed in [23] a seamless multimedia handoff for hierarchical mobile with a novel anticipate buffering scheme. [8] proposed a Predictive Address Reservation with SIP (PAR-SIP) which decreases handoff delay by proactively processing the address allocation and session update using link layer information of wireless networks. [24] introduced application layer techniques to achieve fast handoff for real-time based multimedia traffic in a SIP signaling environment.

In our previous work [16], we introduced fast hierarchical mechanism FHSIP based on PARSIP mechanism [8]. The main contribution of our work is the integrating our anticipated buffering scheme [23] in our fast hierarchical mobile SIP described in [16] to provide a seamless mobility management over heterogeneous networks.

Also, we try to eliminate packet loss and minimise handover latency for mobile multimedia services. At first, a novel buffering scheme is integrated to achieve free packet loss on fast hierarchical mobile sip environment in which we develop a seamless handover scheme called SM-SIP (Seamless Mobility scheme for Mobile SIP environment). SM-SIP can significantly reduce the system signalling cost and handover delay, by proactively processing the address allocation and session updates using link layer information of wireless networks. Also, our performance study shows that SM-SIP reduces efficiently packets loss using an anticipated buffering scheme.

The rest of the paper is organized as follows. Section II provides theoritical backround. Implementation and Simulation details of our proposed solution SM-SIP are described in section III and IV. We finally conclude the paper in section $\mathrm{V}$.

\section{THEORITICAL BACKGROUND}

Mobile IP is designed for mobility management; it causes a high latency and signalling overhead during handover. Therefore, advanced mobility mechanisms improving Mobile SIP are desirable to perform efficient handover. Also, appropriate Quality of Service (QoS) 
support is needed for mobility-enhanced in order to meet mobile user's expectations. For these raisons, our solution SM-SIP uses the Handover Layer 2 information to anticpate buffering scheme. To enhance the fast handover mechanism in SM-SIP we adopte the movement detection defined in PAR-SIP [ 8] where each mobile node starts to search another reachable access point (AP) using active scan as the Signal to Noise Ratio (SNR) value of the current AP falls below the Cell Search Threshold. HMSIP [25] is used to reduce signaling overhead and handoff delay inside the same domain in a SIP environment. It follows the general regional registration approach found in various intra-domains mobility schemes (MIP-RR, HMIPv6) [9] and builds on SIP hierarchical registration. HMSIP defined a new entity responsible for locally handling intra-domain mobility called SIP Mobility Agent (SIPMA). SIPMA is a domain border router enhanced with the functionality of SIP Proxy and SIP Registrar. HMSIP allocates two IP addresses to the Mobile Host (MH), a local address (LA) reflecting the $\mathrm{MH}$ current point of attachment allocated by the serving access router and a global Domain Address (DA) used to uniquely identify the $\mathrm{MH}$ during its roaming inside the same domain, and is allocated by the SIP MA. The SIP MA maintains and manages a data base of soft state mappings between the SIP URI, the DA and the LA for each mobile that roams inside the domain. HMSIP caters for intra-domain mobility and relies on Mobile SIP2 for handling inter-domain mobility.

SM-SIP is based on Hierarchical Mobile SIP to reduce signalling overhead and support seamless handoff in wireless/mobile networks. The regional registration procedure proposed by HMSIP offers low handoff delay, but it remains too high for real time applications which require low handoff. HMSIP can't offer such delays even by eliminating home registration procedure because in addition to time necessary for Link layer handoff, the $\mathrm{MH}$ must detect movements in the network layer (it must discover that it is on a new sub-network using Router Advertisement (RA), which a router periodically broadcasts), obtain a new IP Address, re-configure its own network interface and some network parameters to communicate again, and finally update its location information by sending the SIP REGISTER message to the local registrar. Among the above steps, address allocation takes the most part of the handoff delay. DRCP [27] reduces the address allocation time; a handoff still requires a few hundred milliseconds. Address acquisition becomes then the main cause in degrading the service quality in real-time applications. For this reason and in order to reduce handoff delay at a value suitable for real time applications, we propose to reinforce HMSIP with a Predictive Reservation Address mechanism (PAR) (Fig.2). The regional registration procedure proposed by HMSIP offers low handoff delay, but it remains too high for real time applications which require handoff delay to avoid service degradation. So, to reduce total handover latency, PAR (Predictive Address Reservation) mechanism with HMSIP allows not only reducing handoff delay but also signalling overhead. To obtain

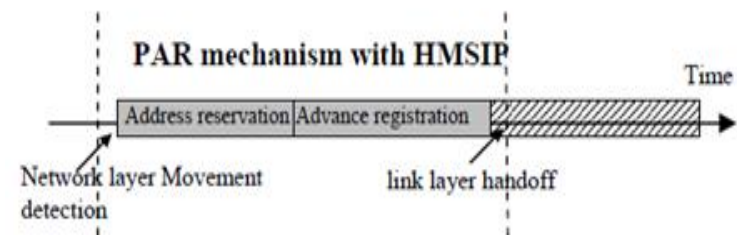

Fig.2. Link layer handover triggering in HMSIP [16]

a new IP address in advance, $\mathrm{MH}$ must forecast its network layer movements before receiving RA (Router Advertisement) from a router. Network layer movements detection is done as follows: When the Signal to Noise Ratio value (SNR) of the current access point (AP) falls below the Cell Search Threshold, the MH starts to search another reachable AP with higher SNR than the current one (active scan). After the selection of such an AP and using an AP list, MH can verify whether the predictive AP belongs to the same access router or to a neighbour one. If the predictive AP belongs to the same access router, a link layer handoff is performed using its address MAC, else MH starts the Address Reservation Process. To the SIP-MA entity is added a data base to manage the domain access routers information. Each AR contains a neighbour AR information table, updated periodically by the SIP MA entity, to allocate a temporary address and to create an AP list for the $\mathrm{MH}$.

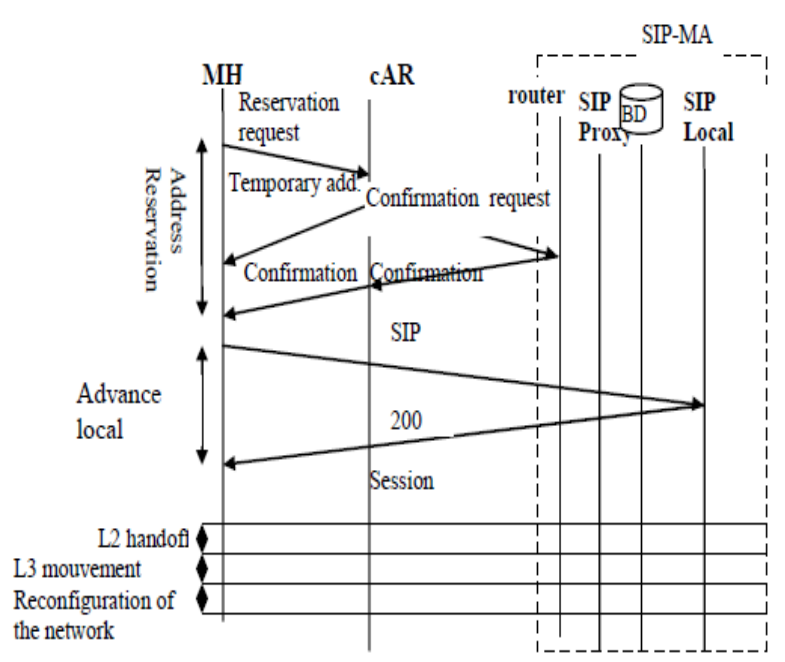

Fig.3. PAR-HMSIP handoff flow [16]

The address reservation process is carried out in the same manner as in [8] with some modifications adapting it to the hierarchical environment. When a MH detects network layer handoff, it sends a reservation request to the current AR, cAR, as shown in the (Fig.3). The cAR sends to $\mathrm{MH}$ a temporary local address, topologically correct for the new sub network, and asks the SIP-MA router to validate this address. The SIP-MA router verifies that the allocated address is not used and replies with a validation message. The cAR then authorizes $\mathrm{MH}$ to use the allocated address. After this, MH sends a SIP REGISTER message to the SIP registrar of the serving SIP-MA and receives the OK message as a reply before the triggering of the link layer handoff. All mobility 
support using SIP proposals, privilege the reestablishment of the ongoing sessions (the SIP re-INVITE message) against the location information update after a handoff. But, in HMSIP environment there is no need to reestablish the ongoing SIP sessions after an intra-domain handoff, because the SIP-MA is informed about the new LA of the MH via the regional registration procedure and is able to Mobile SIP. Redirect the data path to the new location. Therefore there is also no need to re-establish in advance an ongoing session in SM-SIP. This allows reducing the number of procedures to be performed before the link layer handoff triggering and therefore the increase of the probability so that the tasks to be performed before the link layer handoff terminate before the triggering of the link layer handoff.

\section{SM-SIP}

Mobility management with provision of seamless mobility and service continuity is the key topic in mobile network. In this section we will discuss our proposed scheme (SM-SIP).

Our solution tries to provide good QoS performance support for mobile multimedia applications. Signaling overhead and handoff delays are reducing by using fast handover mechanism (combining: Predictive address reservation, handover anticipated) over HMSIP mode. Also, SM-SIP may guarantee seamless multimedia handover as long as the MH moves. But it is still not enough to enhance packet loss over real time and multimedia services. So we propose a integrate data buffering scheme [23] to reduce the amount of buffered user data along with the application requirements by integrating a fast buffering mechanism.

\section{Loss free packet: (Anticipated buffering process using security threshold)}

To minimize loss packet during handover, SM-SIP proposes an anticipate buffering (Fig.4) process with conditional tunnelling. This process enabling the temporary storage of the tunnelled before the handover packets, thus eliminating packet loss occurred during the link layer handover period.

In Handoff mechanism, Signal strength based measurements are considered due to its simplicity [28]. Building on this, we define the two following metrics:

a) LT (Loss threshold): A packet may be considered as lost if it is received with Signal Strength (SS) less than LT.

b) ST (Security threshold): This threshold may be useful to synchronize with the start of Buffering application Handover: related to the initial idea was to start buffering with a broadcast message «Handoff Initiate». However, there may be packets loss before sending this message; we define the security threshold for anticipating the buffering before the signal level deteriorates. Soon as the mobile host receives the signal strength equal to the security threshold, it sends a message Application Control Buffer (RCB) to its old access router. This message acts as initiator of storing packets in the buffer. While the packets are being stored, the old access router sends a copy of these packets until there is a disconnection from the mobile host (reached the loss threshold). Note that the buffering continues until the connection with the new router is established and make a record with the new router.

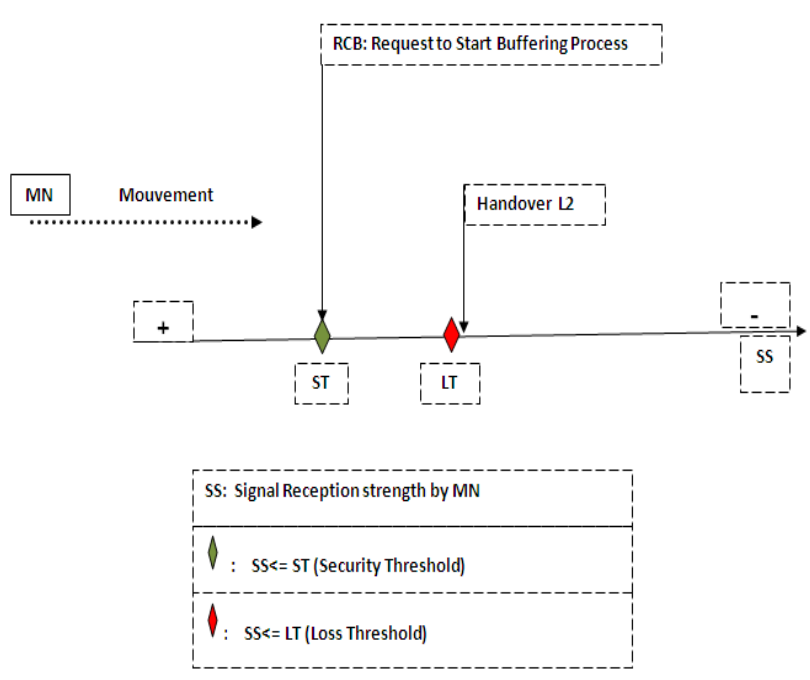

Fig.4. Anticipated Buffering Process [23]

SM-SIP handover (Fig.5) is described as follows:

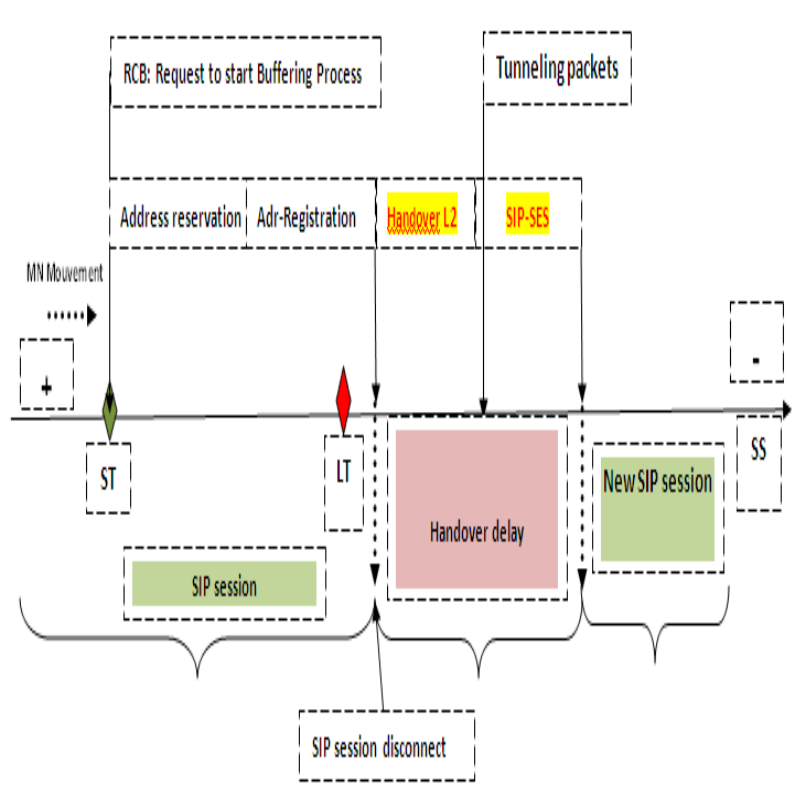

Fig.5. SM-SIP handover

The handoff procedure in mobile SIP environment consists of six subprocedures (Tab.1). Delay for each sub-procedure can be represented with T0 T5 as following: 
Table.1. handover sub-procedures

\begin{tabular}{|l|l|}
\hline T0 & Link layer handoff delay \\
\hline T1 & Movement detection delay \\
\hline T2 & Address allocation delay \\
\hline T3 & Configuration delay \\
\hline T4 & SIP re-INVITE Delay \\
\hline T5 & RTP packet transmission delay \\
\hline
\end{tabular}

SIP_Handoff_Delay $=\mathrm{T} 0+\mathrm{T} 1+\mathrm{T} 2+\mathrm{T} 3+\mathrm{T} 4+\mathrm{T} 5$

With PAR and link layer mechanisms, the delay required for DHCP Transaction and the re-INVITE procedure is not necessary since a $\mathrm{MN}$ proactively performs the address allocation and re-INVITE procedure before handoff. Thus, T2 and T4 values appeared zero. The total handoff delay can be estimated with the following [8]:

$$
\begin{aligned}
\text { SIP-Fast_Handoff_Delay } & =S I P \_H a n d o f f \_D e l a y-T 2-T 4 \\
& =T 0+T 1+T 3+T 5
\end{aligned}
$$

The integration of PAR mechanism in HMSIP (regional registration) reduce efficiently both handover overhead and delay, moreover, Address reservation delay and registration delay values appeared zero(executed in parallel within an existing SIP session). So, SIM-SIP handoff delay can be estimated as follow:

$$
\text { SM-SIP_Handoff_Delay= T0+T6 }
$$

Where T6 = SIP-SES: Reconfiguration time + Time to establish a new SIP session in HMSIP. Note that with SM-SIP (Fig.5):

$$
\mathrm{T} 6<=\mathrm{T} 4+\mathrm{T} 5
$$

\section{Simulation}

\section{Simulation scenario}

$>$ We assume that there is no change in direction while the MH moves inside the overlapping area.

> Simulator: Ns-allinone-2.34

$>$ Network: $1000 * 1000$

$>$ Modulation: DSSS

$>$ Bandwidth: $100 \mathrm{mbps}$

$>$ Traffic generator: video

$>$ Packet rate: 50 packet per second

$>$ Packet size: 512 bytes

> Loss Threshold: 3.41828e-10 Watts

[27] presented a method to simulate sending the video in NS-2. We used this method to simulate the behavior of video transmission to mobile node. Obtaining a trace file can be using as scenario of sending video frames, this trace can be read by the simulator NS-2 (Fig.6). This will correspond to a send data during simulation.

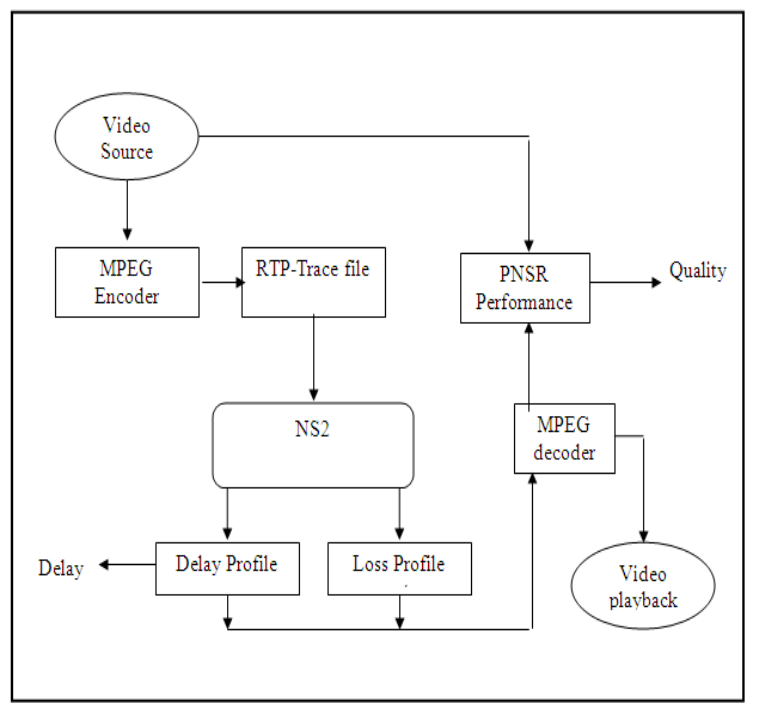

Fig.6. Video simulation model over NS-2

\section{Simulation results}

In this section, we analyze the SM-SIP performance against different mobility solutions. It will be carried out four aspects: handover latency, throughput, jitter and handover loss packets. As we analyzed the performance of our proposed scheme we proved that SM-SIP transmits message faster and efficient than FMIPv6, FHMIPv6 and HMIPv6.

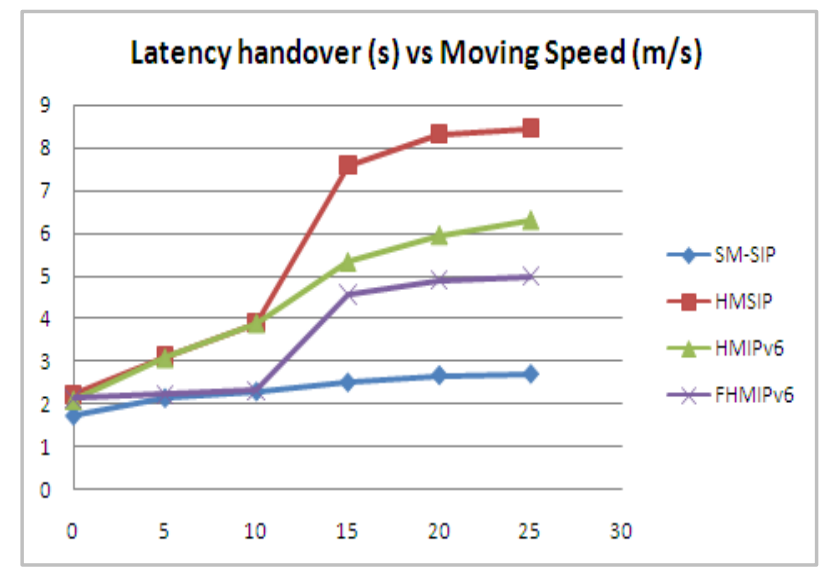

Fig.7. Latency handover vs moving speed

Fig.7 and Fig.8 show the increase in the handover latency and the packet loss due to an increase in moving speed of MHs. As can be seen, SM-SIP approach performs better than FHMIPV6, HMIPv6 and HMSIP in terms of the handover latency and packet loss. Although the SM-SIP (with the integration of thresholds: ST-LT, Using HMSIP, Predictive Address Reservation) is designed to minimize the packet loss and the latency during a handover, a worse performance is observed with respect to HMIPv6 and HMSIP. In contrast, SM-SIP provides a low latency handover (1.04 and $1.75 \mathrm{~s})$. This is due to the fundamental difference between handover registrations and anticipation Procedures in SM-SIP and other procedures. Furthermore, the number of packets lost depends on the moving speed of $\mathrm{MH}$. 


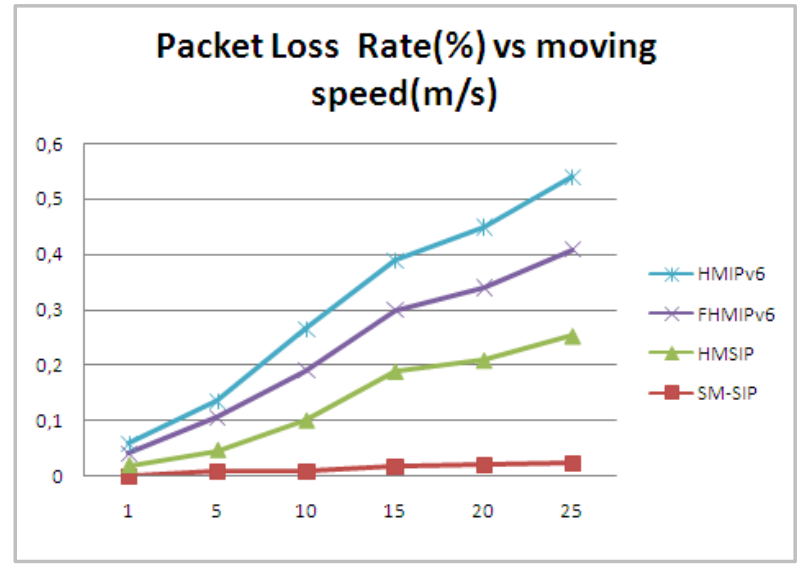

Fig.8. Packet loss Rate (\%) vs moving speed

As seen in the (Fig.8), SM-SIP packets lost rate is between 0 and 0.024 . This means that the packet loss can totally eliminated if we use an anticipated buffering scheme.

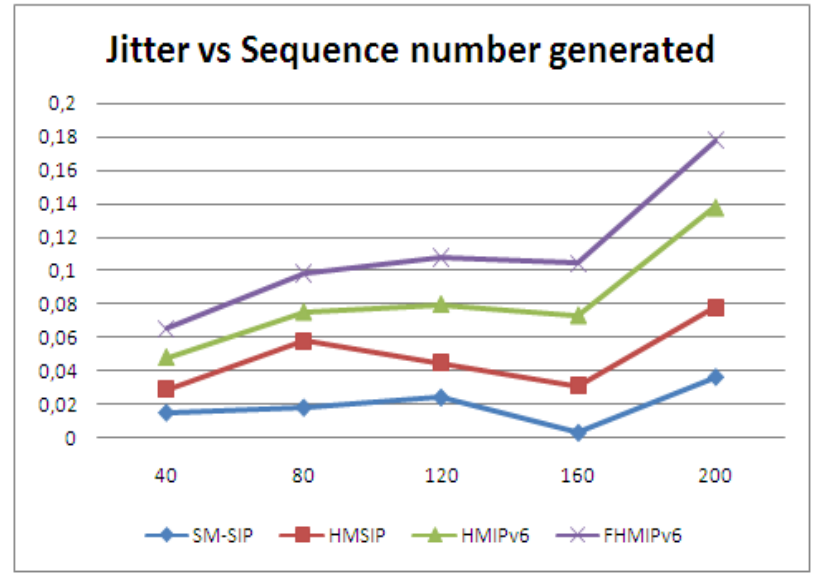

Fig.9. Jitter vs sequence number generated

Fig.9 shows the jitter comparison under video sequence generated. The mean jitter for video traffic is 0.08 and 0.038 s in HMSIP and SM-SIP correspondingly. In the other hand the mean jitter for video traffic is 0.14 and $0.18 \mathrm{~s}$ in HMIPV6 and FHMIPV6 correspondingly. Furthermore, we can see that when the payload is light, there is no loss in SM-SIP for video traffic. We can see also, that there is a difference between HMSIP and the SM-SIP6 under heavy background payload; Payload affects more to the performance of HMIPV6, FHMIPV6 and HMSIP than SM-SIP. When there is heavy background payload, the loss rate of HMIPV6, FHMIPV6 and HMSIP is bigger than that of SM-SIP.

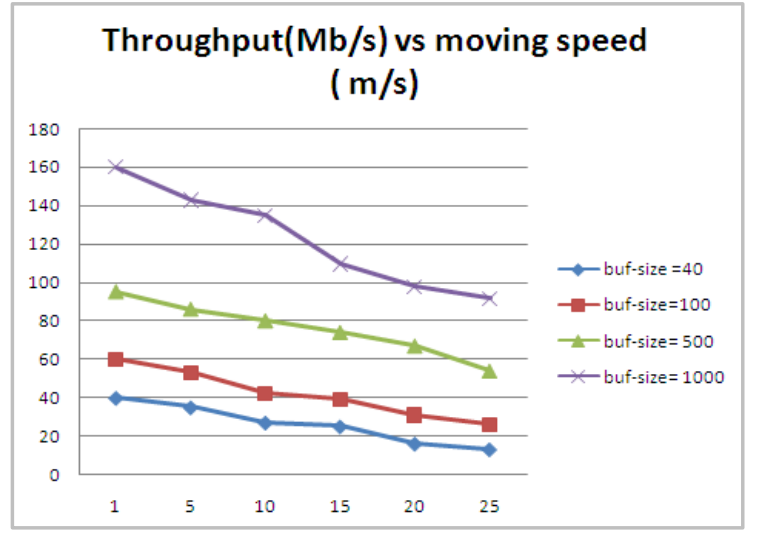

Fig.10. throughput (Mb/s) Vs Moving speed

Fig.10 shows the dependency of the system throughput on buffer size. We allocate different buffer sizes to mobile nodes to see the dependency of the aggregate throughput on the buffer size. Tha average throughput is: $29,25,26$, and 33 for buffer size equal to: 40 packets, $100,500,1000$ correspondingly.

We can say that under small buffer conditions and under video- traffic, a reduction the buffer size choice has a positive effect on the throughput performance. So, we can overcome throughput exceeds the saturation throughput, by allocating the right buffer size. This may not be easy in practice because one does not necessarily know a priori the number of clients in the system or their respective arrival processes. Fortunately, even if one cannot perfectly fit the buffer size to the traffic scenario, not much is lost in term of throughput. As we have seen, a smaller buffer gives identical throughput performance as a large buffer under ten users and more, in which case smaller buffers outperform large buffers.

\section{CONCLUSION}

This paper proposes a handover scheme for achieving mobile multimedia services. The integration of the PAR mechanism with the Hierarchical Mobile SIP reduces significantly the handoff latency. Also, the integration of anticipated buffering reduces significantly the handoff packet losses during the handover process but its integration with the HMSIP environment provides better handoff performance. The paper analyzes the scheme's performance parameters, including the handover latency, throughput, jitter and handover loss packets. As we analyzed the performance of our proposed scheme we proved that SM-SIP transmits message faster and efficient than FMIPv6, FHMIPv6 and HMIPv6. 


\section{REFERENCES}

[1] J.Puttonen, "Mobility Management in Wireless". Published PhD thesis, University of Jyvaskyla, Finland, 2006.

[2] J.Gubbi, R.Buyya, S.Marusic, M.Palaniswami, "Internet of Things (IoT): A vision, architectural elements, and future directions", Future Generation Computer Systems, Vol.29, P.1645-1660, 2013

[3] M.Ulema, J.M.Nogueira, and B.Kozbe "Management of Wireless Ad Hoc Networks andWireless Sensor Networks", Journal of Network and Systems Management, Vol. 14, No. 3, 200677.

[4] K.Yang, Y.Wu, H.Chen, "QoS-aware routing in emerging heterogeneous wireless networks, Quality-of-ServiceBased Routing Algorithms for Heterogeneous Networks" IEEE Communications Magazine, 2007.

[5] H.Fathi, S.Chakraborty and R.Prasad, "Mobility Management for VoIP in 3G System: Evaluation of LowLatency Handover Schemes", IEEE Wireless Communication magazine, Vol. 12, Issue 2, pp. 96-104, 2005.

[6] C.Perkins," IP Mobility Support forIPv4,"RFC3344, IETF", August2002, http://www.ietf.org/rfc/rfc3344.txt?number=3344/ (Accessed 20 March 2011).

[7] Y.Leu, "A novel network mobility handoff scheme using SIP and SCTP for multimedia applications", Journal of Network and Computer Applications, vol. 32, pp. 1073$1091,2009$.

[8] W.M.Kim, K.Kim, C.Lee.Yu and L.Link, "Layer Assisted Mobility Support Using SIP for Real-time Multimedia Communicationsé" ACM MobiWac. Philadelphia, PA, U.S.A, 2004.

[9] M.Tao, H. Yu, "A Smooth Handover Scheme for FastMoving Users in Mobile IPv6 Networks", WIRELESS PERSONAL COMMUNICATIONS, Vol.60, No 4, pp. 649664, 2010.

[10] L.J.HANG, P.Samuel, "Evaluating the Performance of Fast Handover for Hierarchical MIPv6 in Cellular Networks", JOURNAL OF NETWORKS, Vol. 3, No. 6, pp. 36-43, 2008.

[11] W. Xiaonan, Q. Huanyan, "Research on all-IP communication between wireless sensor networks and IPv6 networks", Computer Standards \& Interfaces, vol 35, pp 403-414, 2013.

[12] W.Xiaonan, Z.Shan, "All-IP communication between wireless sensor networks and IPv6 networks based on location information" Computer Standards \& Interfaces, vol. 35, pp 65-77, 2013.

[13] A.Berguiga, H.Youssef, "A Fast Handover Scheme for Proxy-based Mobility in Wireless Sensor Networks" MobiWac'12, October 21-22, Paphos, Cyprus, 2012.

[14] N.Banerjee, W.Wu, K.Basu, S.K.Das, "Analysis of SIPbased mobility management in 4G wireless networks" Computer Communications", vol. 27, pp.697-707, 2004.

[15] N.Banerjee, A. Acharya and S.K.Das, "Seamless SIPbased mobility for multimedia applications," IEEE Network, vol. 20, no. 2, 2006.

[16] F.Chahbour, N.Nouali, K.Zeraoulia, "Fast Handoff for Hierarchical Mobile SIP network ",The Third World Enformatika Conference, WEC'05, April 27-29, Istanbul, Turkey, 2005.

[17] Y.Leu, "A novel network mobility handoff scheme using SIP and SCTP for multimedia applications", Journal of Network and Computer Applications Vol. 32, pp. 10731091, 2009.
[18] M.Alnas, I.Awan, R.D.W.Holton, "Performance Evaluation of Fast Handover in Mobile IPv6 Based on Link-Layer Information", the Journal of Systems and Software 83 1644-1650, 2010.

[19] L.J.Zhang, S.Pierre, “Intelligent Fast Handover Scheme for Mobile IPv6-based Wireless Local Area" Networks IJCSNS International Journal of Computer Science and Network 60 Security, VOL.9 No.8, August 2009

[20] M.Torrent, X.Perez-Costa, S.Sallent-Ribes,"A Performance Study of Fast Handovers for Mobile IPv6", 28th Annual IEEE International Conference on Local Computer Networks (LCN'03), 2003.

[21] Yao.M, and Chen.Y" An Enhanced Buffer Management Scheme for Fast Handover Protocol", Proceedings of the 24th International Conference on Distributed Computing Systems Workshops (ICDCSW’04), 2004.

[22] S.Ryu, K.Lee, Y.Mun, "Optimized fast handover scheme in Mobile IPv6 networks to support mobile users for cloud computing J Supercomput, 2010

[23] K.Zeraoulia, N.Badache, "Seamless Multimedia Handoff for Hierarchical Mobile IPv6" The Seventh International Conference on Wireless and Mobile Communications ICWMC 2011 June 19-24, Luxembourg, 2011.

[24] A.Dutta, S.Madhani, W.Chen," Fast-handoff Schemes for Application Layer Mobility Management", 15th IEEE International Symposium on Personal, Indoor and Mobile Radio Communications, PIMRC 2004, 2004.

[25] N.C.Chang, T.Mai, L.H.Chang, "HMSIP: Hierarchical Multicast SIP Protocol for streaming multimedia in wireless networks", IEEE International Conference on Networking, Sensing and Control, Taipei, Taiwan, March 21-23, 2004

[26] A.Barnawi,A.H.Altalhi,M.R.Qureshi,A.I.Khan,"Evaluation of IP Multimedia Subsystem based Mobile Mass Examination System" I. J. Computer Network and Information Security, 2012, 4, 1-12

[27] S.Linck, E.Mory, J.Bourgeois, E.Dedu,"Video quality estimation of DCCP streaming over wireless networks", 14th Euromicro International Conference on Parallel, Distributed, and Network-Based Processing, February 15th - 17th, Montbéliard-Sochaux - France, 2006.

[28] M.N.Vikas,K.N.Keshava,R.K.Prabhas,I.Hameem-Shanava, "Efficient Hand off using Fuzzy and Simulated Annealing" I. J. Computer Network and Information Security, 2012, 1, $17-23$

[29] A.Bhuvaneswari, E.G.D.Prakash Raj, "An Overview of Vertical Handoff Decision Making Algorithms" I. J. Computer Network and Information Security, 2012, 9, 5562.

\section{Authors' Profiles}

Zeraoulia Khaled He graduated in 2002 as Dipl. Eng, and got his Master in 2005 from Electrical Engineering and computer science (USTHB), with a thesis on "video transmission over wireless networks". Since 2008 he is PhD student in this faculty, working on a thesis entitled "Quality of Services in wireless multimedia mobile communications".

Nadjib Badache received the engineer degree in computer science from the University of Constantine, Algeria, in 1978 and the master's and $\mathrm{PhD}$ degrees from the University of Sciences and Technology Houari Boummediene (USTHB), 
Algiers, Algeria, in 1982 and 1998, respectively. He is currently a professor in the Computer Science Department, USTHB, where he is also the head of CERSIT. His research interests are distributed mobile systems, mobile ad hoc networks, and security.

How to cite this paper: Khaled Zeraoulia, Nadjib Badache,"SM-SIP: Seamless Mobility Management for Heterogeneous Wireless Networks", IJCNIS, vol.6, no.9, pp.58-65, 2014. DOI: 10.5815/ijcnis.2014.09.08 